\title{
Perspectivas da deficiência física no idoso: vulnerabilidades em saúde
}

\section{Outlooks on disability in older adults: health vulnerabilities \\ Perspectivas sobre la discapacidad en los ancianos: la vulnerabilidad de la salud}

\author{
Juliana Balbinot Reis Girondil; Silvia Maria Azevedo dos Santos"II; Simone Cristine dos Santos Nothaft ${ }^{\text {III }}$
}

\begin{abstract}
RESUMO: Pesquisa qualitativa e descritiva. Objetivou investigar as percepções de idosos, gestores e profissionais de saúde sobre as perspectivas da deficiência física no idoso. Foram realizadas 18 entrevistas com idosos, por meio de roteiro semiestruturado, e aplicados questionários a 17 gestores e três profissionais de saúde, de junho a dezembro de 2010, em Florianópolis - Santa Catarina. A análise dos dados, através da hermenêutica dialética, levou à apreensão dos elementos constitutivos das três categorias: O impacto da deficiência física na qualidade de vida do idoso; as redes de suporte social; a naturalização da deficiência no processo de envelhecimento. Conclui-se que o impacto da deficiência afeta a qualidade de vida dos idosos; embora profissionais e gestores a considerem como um processo natural do envelhecimento. Em relação aos cuidados, há uma frágil rede de apoio, predomínio do modelo biomédico, deficiência na formação acadêmica e na instrumentalização das equipes de estratégia de saúde.
\end{abstract}

Palavras-Chave: Envelhecimento; idoso; vulnerabilidade social; atenção primária à saúde.

ABSTRACT: This qualitative, descriptive study investigated perceptions among older adults, managers and health professionals as to the outlook for disability in the elderly. Semi-structured interviews were conducted of 18 older adults and questionnaires were applied to 17 managers and three health professionals, between June and December 2010 in Florianópolis, Santa Catarina, Brazil. Data analysis through dialectic hermeneutics apprehended the constituent components of three categories: the impact of disability on older adults' quality of life; social support networks; and naturalization of disability in the aging process. It was concluded that the impact of disability affects older adults' quality of life, while health professionals and managers consider it a natural part of the aging process. As regards care, there is a fragile support network, the biomedical model predominates, and Family Health Strategy teams' academic training and instruments are deficient.

Keywords: Aging; elderly; social vulnerability; primary health care.

RESUMEN: Investigación cualitativa y descriptiva cuyo objetivo fue investigar las percepciones de los ancianos, gestores y profesionales de la salud sobre las perspectivas de la discapacidad física en los ancianos. Se llevaron a cabo 18 entrevistas con ancianos a través de entrevista semiestructurada y cuestionarios a 17 directivos y 03 profesionales de la salud, de junio a diciembre de 2010 en Florianópolis, Santa Catarina. El análisis de los datos, a través de la hermenéutica dialéctica, llevó a considerar los componentes de tres categorías: el impacto de la discapacidad física en la calidad de vida del anciano; las redes de apoyo social; la naturalización de la discapacidad en el proceso de envejecimiento. Se concluye que el impacto de la discapacidad afecta la calidad de vida de los ancianos; aunque los profesionales y directivos la consideren como un proceso natural del envejecimiento. En cuanto a la atención, hay una frágil red de apoyo, con predominio del modelo biomédico, deficiencia en la formación académica y la instrumentalización de los equipos de la Estrategia de Salud.

Palabras Clave: Envejecimiento; anciano; vulnerabilidad social; atención primaria de salud.

\section{INTRODUÇÃO}

O envelhecimento populacional constitui um dos maiores desafios para a saúde pública contemporânea, especialmente em países em desenvolvimento, onde esse fenômeno ocorre em ambiente de pobreza e de grande desigualdade social ${ }^{1}$. Além disso, evidencia-se o despreparo da saúde pública mundial frente ao aumento rápido e contínuo da longevidade humana ${ }^{2}$.
O processo do envelhecer delimita mudanças de ordem biológica, psicológica e social. E, em grande parte destas populações pouco se têm feito para que as limitações não causem deficiências. É possível detectar que a deficiência pode ter sua origem pela falta e/ou dificuldade de acesso ao cuidado. As condições de saúde da população idosa podem ser determinadas

IDoutora em Enfermagem. Professora do Curso de Graduação em Enfermagem e do Programa de Pós-Graduação em Enfermagem da Universidade Federal de Santa Catarina. Membro do Grupo de Estudos sobre Cuidados de Saúde de Pessoas Idosas. Florianópolis, Santa Catarina, Brasil. E-mail: juliana. balbinot@ufsc.br.

IIEnfermeira. Doutora em Educação. Docente do Departamento e do Programa de Pós-Graduação em Enfermagem da Universidade Federal de Santa Catarina. Líder do Grupo de Estudos sobre Cuidados de Saúde de Pessoas Idosas. Florianópolis, Santa Catarina, Brasil. E-mail: azevedosms@gmail.com. IIIEnfermeira. Mestranda do Programa de Pós-Graduação da Universidade Comunitária da Região de Chapecó. Docente no Departamento de Enfermagem da Universidade do Estado de Santa Catarina. Membro do Núcleo de Pesquisa e Estudos em Enfermagem, Quotidiano, Imaginário e Saúde. Santa Catarina, Brasil. E-mail: E-mail: nothaft@hotmail.com. 
pelos seus perfis de morbidade e mortalidade, presença de déficits físicos e cognitivos e a utilização dos serviços de saúde, dentre outros indicadores mais específicos ${ }^{1}$. Uma das condições que pode vir a se instalar nos idosos são as deficiências físicas.

Deficiência é o termo usado para definir a ausência ou a disfunção de uma estrutura psíquica, fisiológica ou anatômica do ser humano ${ }^{3}$. No Brasil, considera-se pessoa com deficiência física aquela que apresenta alteração completa ou parcial de um ou mais segmentos do corpo humano, acarretando o comprometimento da função física, apresentando-se sob a forma de paraplegia, paraparesia, monoplegia, monoparesia, tetraplegia, tetraparesia, triplegia, triparesia, hemiplegia, hemiparesia, ostomia, amputação ou ausência de membro, paralisia cerebral, nanismo, membros com deformidade congênita ou adquirida, exceto as deformidades estéticas e as que não produzam dificuldades para o desempenho de funções ${ }^{4}$.

A Organização Mundial de Saúde (OMS) estima que cerca de $10 \%$ da população de qualquer país em tempo de paz é portadora de algum tipo de deficiência. Com base nesse percentual, estima-se que no Brasil existam 16 milhões de pessoas portadoras de deficiência ${ }^{5}$. Essas duas situações - ser idoso e ter uma deficiência - por si mesmas já constituem situações de vulnerabilidade. Apesar de o modelo de atendimento ao idoso com deficiência preconizar a interdisciplinaridade, na prática ainda não foi estabelecido plenamente. Além disso, esse modelo normalmente não é discutido na academia ou valorizado pelas políticas de saúde e, conseqüentemente, os profissionais de saúde encontram muitas dificuldades em implementá-lo.

Frente ao exposto, o adequado enfrentamento das demandas causadas pelo envelhecimento populacional permitirá a efetivação de um cuidado integral aos idosos, sintonizado com as práticas de promoção da saúde e prevenção de agravos. Os cuidados aos idosos implicam em ofertar serviços que propiciem o acesso e o acolhimento de maneira ordenada e adequada, respeitando as suas limitações. Nesse processo, a atenção primária à saúde deve configurar a porta de entrada dos idosos ao sistema de saúde, onde será prestada uma assistência de qualidade e de resolutividade.

Tal constatação remete ao fato de que profissionais de saúde, gestores do sistema público de saúde e a sociedade como um todo devem despertar para a necessidade de novas estratégias de cuidar destes idosos. As estratégias de promoção da saúde devem ser consideradas no sentido de oportunizar a igualdade de recursos para que os idosos com deficiência física possam ter apoio, acesso à informação, habilidades para a vida e oportunidades para fazer escolhas saudáveis. Através de novos estudos, legislações e ações comunitárias é que a história desses idosos será delineada.

Por conseguinte, este estudo teve como objetivo: investigar as percepções de idosos, gestores e profis- sionais de saúde da atenção primária à saúde sobre as perspectivas da deficiência física no idoso.

\section{RevisÃo de LITERATURA}

A sustentação teórica deste estudo foi pautada nos princípios do Sistema Único de Saúde (SUS) ${ }^{5}$ e na Política Nacional da Pessoa Portadora de Deficiência Física ${ }^{6}$.

O SUS segue a mesma doutrina e os mesmos princípios organizativos em todo o território nacional, sob a responsabilidade das três esferas autônomas de governo: federal, estadual e municipal. São seus princípios: a Universalidade, a Equidade e a Integralidade. Na abordagem ao idoso, o princípio da Universalidade consiste em assegurar que todos os idosos tenham direito ao atendimento à saúde, já que esta é um direito de todo cidadão e dever do governo. A Equidade, por sua vez, garante que todo cidadão é igual perante o sistema, por isso esse idoso deverá ser atendido conforme as suas necessidades. Isso é importante, pois os serviços de saúde devem considerar que em cada população existem grupos de idosos que vivem de forma diferenciada, possuem problemas específicos, diferentes formas de viver e de satisfazer suas necessidades 5 .

Dessa forma, os serviços de saúde poderão auxiliar na diminuição das desigualdades existentes no país. Ao passo que a Integralidade consiste em um conjunto de ações de saúde específicas ao idoso, que devem ser articuladas e voltadas para prevenção de doenças e a cura 5 .

Os serviços de saúde devem funcionar na perspectiva de atender esse indivíduo como um ser integral, holístico. O foco do atendimento deve ser voltado para a saúde das pessoas e não somente para as suas doenças. Desse modo, será possível desenvolver ações integradas para promoção, prevenção de doenças e recuperação da saúde. Vale ressaltar que a atenção primária em saúde tem como um dos seus fundamentos possibilitar o acesso universal e contínuo a serviços de saúde de qualidade, reafirmando os princípios básicos do SUS.

O resgate desses princípios é pertinente, tendo em vista que é neste sistema de cuidado que os idosos com deficiência física estão inseridos, mesmo que marginalmente ${ }^{5,6}$. Como uma das premissas do SUS é possibilitar o acesso dessas pessoas ao sistema de saúde, deve-se pensar que para os idosos com deficiência essa acessibilidade é um processo. E tal processo deve oportunizar que essas pessoas participem da vida em sociedade, que sejam independentes, bem como que possam ter garantido o princípio de igualdade, principalmente nas questões relativas à sua vida e saúde ${ }^{6}$.

Nesse contexto, ao longo dos anos, a legislação nacional foi sendo aprimorada, através de leis, portarias e decretos, a fim de efetivar a Política Nacional da Pes- 
soa Portadora de Deficiência Física. Esta política é um instrumento que orienta as ações do setor saúde voltadas para esse segmento populacional que objetiva prioritariamente reabilitar a pessoa portadora de deficiência na sua capacidade funcional e no seu desempenho humano. Apesar de o Brasil ser um dos poucos países no mundo a ter uma legislação específica, ainda se verifica que as pessoas com deficiência são bastante excluídas da sociedade? Percebe-se que, no âmbito da deficiência em idosos, a discussão ainda é bastante insipiente.

\section{Metodologia}

Estudo qualitativo, do tipo descritivo, com coleta dos dados realizada entre junho e dezembro de 2010. Para a seleção do cenário da pesquisa, foi realizado um levantamento quantitativo de idosos com idade igual ou superior a 60 anos, por meio do Setor de Planejamento da Secretaria Municipal de Saúde de Florianópolis, Santa Catarina, Brasil. Esse município possui uma população de 421.240 residentes, sendo que a população acima de 60 anos totaliza 48.894 pessoas $(11,5 \%)^{8}$.

No âmbito da saúde, o município está dividido em cinco distritos sanitários (DS), que abrangem 48 centros de saúde. Desse total, foram selecionados dois DS localizados: no Continente e no Sul de Florianópolis. A escolha por estas regiões se deu em virtude das diferenças geográficas, sociais, epidemiológicas e culturais. Nesses distritos, selecionou-se um centro de saúde de cada, utilizando como critério aqueles que apresentaram maior contingente de idosos. Assim, foram escolhidos o Centro de Saúde 1 (CS1) - pertencente ao DS Continente -que possui em sua área de abrangência 565 idosos (7,44\% da população total) e o Centro de Saúde 2 (CS2) - pertencente ao DS Sul - que possui 126 (7,49\% da população total $)^{8}$.

Para o desenvolvimento do estudo, foram convidados a participar os idosos com deficiência física que utilizam os CS selecionados, os profissionais de saúde desses centros de saúde e os gestores da Secretaria Municipal de Saúde de Florianópolis. Participaram da pesquisa 18 idosos que atenderam aos critérios de inclusão: ter deficiência física congênita ou adquirida há pelo menos 1 ano, idade igual ou superior a 60 anos, ausência de alterações cognitivas e/ou mentais graves (verificada através da aplicação do Miniexame do Estado Mental - MEEM), que aceitaram participar da pesquisa e assinaram o Termo de Consentimento Livre e Esclarecido. Para a localização desses sujeitos, foi realizada busca ativa pela equipe da estratégia saúde da família (ESF) e agentes comunitários de saúde (ACS), mediante um instrumento elaborado por uma das autoras deste artigo. Para a coleta de dados foi utilizada a entrevista semiestruturada. Todas as entrevistas foram gravadas em áudio e transcritas pela pesquisadora.
Para representar os profissionais de saúde, participaram três sujeitos, sendo dois enfermeiros e um médico. Foram critérios de inclusão: estar vinculado à ESF, ocupar este cargo há mais de um ano, aceitar participar da pesquisa e assinar o Termo de Consentimento Livre e Esclarecido. A escolha por estas duas categorias profissionais se deu em virtude deles integrarem a composição mínima da equipe de ESF.

Para representar os gestores, participaram do estudo 17 pessoas que atenderam aos critérios de inclusão: ocupar cargo de gestor em qualquer nível da estrutura hierárquica (cargo de gerência, diretoria, assessoria, chefia de departamento ou coordenador local de saúde), estar nesse cargo há mais de um ano, aceitar participar da pesquisa e assinar o Termo de Consentimento Livre e Esclarecido.

Para coleta de dados dos gestores e profissionais de saúde utilizou-se um questionário desenvolvido pela pesquisadora. Esse instrumento era autoaplicável e, por isso, a sua entrega foi feita concomitantemente ao agendamento de uma data para seu recolhimento.

Para resguardar o sigilo e o anonimato dos sujeitos da pesquisa, optou-se por identificá-los como usuário (U), para representar os idosos; P para representar os profissionais de saúde e $\mathrm{G}$ para representar os gestores em saúde. No que se refere aos gestores, para cada esfera de atuação utilizou-se uma especificação: GL (gestor local), GR (gestor regional) e GC (gestor central). Como técnica de análise de dados foi utilizada a hermenêutica dialética.

O estudo foi aprovado pelo Comitê de Ética em Pesquisa da Universidade Federal de Santa Catarina, sob protocolo $n^{\circ}$ 695/10

\section{Resultados e Discussão}

\section{Perfil dos participantes}

Quanto às características sociodemográficas, em relação à população idosa estudada, a média de idade foi de 76 anos, com predomínio do sexo feminino. A média de escolaridade dos entrevistados foi de 2,3 anos de estudo, a renda familiar mensal média oscilou entre um e três salários mínimos (12 idosos) na época de realização deste estudo. Quanto ao estado civil, houve a prevalência de casados, representados por oito idosos, seguida de viúvos. Em relação à composição familiar, houve maior distribuição de idosos que residem com o cônjuge (seis), seguido por aqueles que residem com cônjuge e filhos (três idosos). Quanto ao tipo de deficiência houve predomínio da deficiência adquirida (17 idosos).

Em relação aos gestores: cinco eram de nível central, 10 de nível regional e dois de nível local. A média de idade foi de 38, em relação ao gênero, houve prevalência do sexo feminino. Quanto à escolaridade, cinco possuíam somente graduação, três, mestrado e nove, especialização. A categoria profissional de maior 
destaque foi a de enfermeiros, seguida por médicos, psicólogo e dentista. $\bigcirc$ tempo de atuação profissional foi de 13 anos. O tempo de trabalho na instituição foi de 8,7 anos e na ocupação do cargo atual foi de 4,2 anos.

Em relação aos profissionais de saúde, um era médico e dois enfermeiros. A média de idade foi de 38,6 anos, houve prevalência do sexo feminino. Quanto à escolaridade, um possuía especialização, os demais, apenas graduação. $\mathrm{O}$ tempo de atuação profissional foi de 10,6 anos. O tempo de trabalho na instituição e na ocupação do cargo atual foi de 5 anos.

\section{Categorias do estudo}

A análise dos dados possibilitou a formação de um eixo temático sob a forma de três categorias, quais sejam: $\bigcirc$ impacto da deficiência física na qualidade de vida do idoso; As redes de suporte social; A naturalização da deficiência no processo de envelhecimento.

$\mathrm{Na}$ categoria $\mathrm{O}$ impacto da deficiência física na qualidade de vida do idoso se evidencia de que forma a deficiência física interfere na autonomia, dependência/ independência, nas limitações, no desenvolvimento/ realização pessoal dos idosos e de que forma repercutem na qualidade de vida dos mesmos.

Em relação aos idosos que demonstraram relacionar a deficiência física a seu modo de vida, fica evidente que se deve tentar manter o idoso o máximo possível, junto a seus familiares e à comunidade, com independência funcional e autonomia9. A saúde, no contexto do idoso, seria, em um sentido amplo, o resultado do equilíbrio entre as várias dimensões da capacidade funcional do idoso, sem necessariamente significar ausência de problemas em todas as dimensões ${ }^{10}$. O que pode ser observado nas declarações:

Eu vivo bem com isso. Aceito e tem que aceitar. É uma pena porque perdi um pouco a liberdade, eu andava muito antes [...]. (U7)

Minha vida é normal, boa. Não posso fazer quase nada, mas sou feliz. Vivo tranquila [...]. (U10)

Esses achados vêm ao encontro do pressuposto de que a identidade não é um dado biológico, mas uma construção histórica. Repensar o passado, considerando um indivíduo como sujeito, é parte fundamental da construção da dimensão simbólica do processo social ${ }^{11}$.

No entanto, o conviver com a dependência pode, em alguns momentos, promover desânimo dos familiares e do próprio idoso ${ }^{12}$.

Minha vida é uma tristeza! É só nessa cama. Não posso fazer nada [...] mas a gente tem que se conformar com o que Deus dá para a gente. Preciso de ajuda para tudo, me limpar, tomar banho, comer [...]. (U8)

A minha vida agora praticamente acabou [...] Antes eu fazia de tudo. Eu queria tanto andar, mesmo que fosse um pouquinho só. Não depender dos outros, mas não dá. É duro [...]. (U11)
Apesar de as experiências de incapacidade fazerem parte do modo de viver dessas pessoas, elas precisam ter habilidades para superar suas dificuldades e realizar suas ações no mundo ${ }^{13}$. Há que se refletir sobre o fato de que a interdependência e o cuidado são algo necessário não apenas diante da condição de deficiência, mas em vários momentos da vida das pessoas.

Alguns estudos apontam que a pessoa com dependência se sente inútil por necessitar de outros para realização de suas atividades básicas cotidianas: esta situação causa desgaste em quem cuida e em quem é cuidado $^{14,15}$.

Nessa categoria, pretende-se evidenciar que o universo do idoso com deficiência física é amplo, muito além do que se investiga quando a deficiência é apenas contabilizada como um grave comprometimento da capacidade motora. Mudanças comportamentais e sociais, especialmente no âmbito da saúde, podem permitir que vários ajustes sejam implementados, a fim de que estas restrições físicas e corporais não sejam um empecilho na vida desses idosos.

A categoria As redes de suporte social constitui a soma de todas as relações que o idoso considera como significativas na sua vivência com deficiência física e que lhe conferem apoio social, podendo também contribuir para o seu cuidado de saúde. Nessa rede estão inclusos: familiares, cuidadores, amigos, vizinhos, relações comunitárias, serviços e profissionais de saúde. $\mathrm{O}$ suporte social abrange políticas e redes de apoio sociais, tem como objetivo contribuir para o bem-estar das pessoas, principalmente no caso de idosos com deficiência física.

A minha vida, com a graça de Deus éboa. Muito boa, com o auxilio do meu anjo (refere-se à cuidadora) [...]. (U4)

A minha vida é boa. Deus é muito bom comigo. Deume esse marido, meus filhos... [...]. (U5)

Ficou evidente que os idosos vislumbraram uma valorização da vida e da convivência familiar após a instalação da deficiência, podendo contar com o apoio familiar. A rede de suporte familiar é quem dá sustentação para os cuidados e demandas decorrentes da deficiência física. Também se constatou que o cuidador é uma figura importante na manutenção da independência desses idosos.

Esse achado também esteve presente num estudo $^{14}$ em que a família caracteriza-se pela dinâmica das relações, perseverando para a humanização e socialização, sendo o alicerce no apoio e nos cuidados dispensados aos idosos.

Em contraponto a esses achados, seguem colocações que demonstram a insatisfação do atendimento das necessidades desses idosos em relação ao sistema público de saúde.

[...] sempre que a gente precisa de médico e outras coisas ainda não consegue. É duro [...]. (U11) 
[...] o posto é muito fraquinho, só tem aqueles remédios fraquinhos [...] o que a gente precisa não tem [...]. (U5)

$\mathrm{O}$ aumento de doenças crônico-degenerativas e suas sequelas ampliam o desafio de cuidar desses idosos ${ }^{15}$, por conta da questão da dependência funcional. Neste contexto, as ações de saúde hoje estão focadas na questão da promoção de saúde e prevenção de agravos, buscandose o envelhecimento saudável e sustentável.

Assim, para a efetivação do cuidado ao idoso será necessária a transformação do paradigma hegemôni$\mathrm{co}^{16}$ da medicina, para um modelo socioambiental ${ }^{17}$, que considere as doenças a partir de uma perspectiva mais abrangente, analisando vários outros processos envolvidos no perfil de saúde das pessoas idosas.

Os sujeitos da pesquisa relataram ser o médico a referência para eles enquanto o cuidador de sua saúde, evidenciando que a atenção à saúde é centrada nele e há dificuldade em desvincular a saúde da figura deste trabalhador, reafirmando a hegemonia do modelo biomédico ${ }^{18}$. Essa limitação acaba por reforçar o modelo biomédico ${ }^{19}$, centrado na doença, na incapacidade e suas limitações.

Eu gosto do posto porque os médicos vêm bastante na casa da gente [...]. (U15)

A única coisa que a gente é mais ou menos atendido ali no posto é o atendimento médico [...]. (U11)

No entanto, os usuários reconhecem a atuação do enfermeiro. Evidenciam que esse profissional tem atuado com ações de promoção da saúde, prevenção de agravos e reabilitação do idoso ${ }^{20}$ com deficiência física.

A enfermeira vem aqui para medir a pressão e dar a vacina da gripe [...]. (U14)

A enfermeira vinha aqui em casa para fazer o meu curativo [...]. (U5)

Como integrante da ESF, o enfermeiro tem uma visão mais ampliada do idoso, bem como dos cuidados de enfermagem para com ele nas várias instâncias, incluindo: a biológica, a psicossocial, a psicológica, dentre outras. $\mathrm{O}$ enfermeiro interage com a equipe interdisciplinar, desempenhando atividades de promoção à saúde e de proteção a esse idoso ${ }^{21}$, sendo verdadeiramente reconhecido no seu papel.

A categoria A naturalização da deficiência no processo de envelhecimento compreende as várias concepções que permeiam a deficiência física como evento pertinente ao processo de envelhecimento. Inclui a forma como os profissionais de saúde são instrumentalizados para a prática de cuidado da saúde do idoso, além das dinâmicas e interfaces da atenção primária em saúde no município estudado.

De modo geral, os gestores e profissionais de saúde entrevistados possuem uma visão de que a deficiência faz parte do processo de envelhecimento, sendo considerada então, uma ocorrência natural.
[...] o idoso com deficiência física é um indivíduo que apresenta limitações biológicas, fisiológicas desgastantes provocadas pelo avanço cronológico das estruturas orgânicas, e/ou por traumas que acarretam em perda dos sentidos, inserido em contextos isolados e coletivos que refletem em suas condições de vida [...]. (GRS5)

[...] o idoso com deficiência física não consegue haver as suas necessidades e depende de terceiros para se locomover, ou realizar outras atividades como: banho, alimentação, e outros [...]. (GC4)

Muitas dessas concepções foram sendo adquiridos a partir da própria formação acadêmica, atreladas à falta de instrumentalização e de educação continuada na área do envelhecimento.

Este fato foi encontrado também em outros trabalhos científicos que apontam que a formação na área de saúde do idoso se depara com alguns entraves, tais como: a escassez de conteúdo gerontogeriátrico nos currículos ${ }^{22}$, a falta de campos específicos para esta prática ${ }^{23}$ e a inexperiência do corpo docente.

As vulnerabilidades constituem um conjunto de situações que podem tornar o idoso frágil. De acordo com a Organização Mundial de Saúde (OMS), os idosos considerados frágeis são aqueles que possuem uma ou mais das seguintes características: idade superior a 80 anos; residem sozinhos; mulheres (especialmente solteiras e viúvas); as que residem em instituições; os isolados socialmente; os que não têm filhos; os que têm limitações severas ou incapacidades; casais em que um dos cônjuges é incapacitado ou está doente; aqueles com recursos escassos ${ }^{24,25}$.

Assim, pode-se dizer que a deficiência física constitui uma situação de vulnerabilidade à qual o idoso está exposto, podendo vir a constituir uma situação de dependência. No entanto, o idoso pode apresentar alguma deficiência física sem ser dependente de cuidados de outrem. Várias condições e situações permeiam essas conjunturas, devendo ser avaliadas e consideradas no processo de cuidado.

\section{Conclusão}

Refletir sobre os temas envelhecimento, dependência e suas vulnerabilidades é repensar sobre este grande desafio imposto à sociedade pelas demandas do envelhecimento populacional, fenômeno brasileiro e mundial. Neste novo cenário, a previsão é que aumente a procura pelos serviços de saúde pública.

Há que se entender que a dependência não equivale à velhice, e que a sua prevenção perpassa o campo da saúde. Inclui também a esfera socioeconômica e cultural na qual esse idoso está inserido. Estratégias como a perpetuação de hábitos saudáveis por toda a população estendendo-se até o acesso aos serviços básicos de saúde, educação, cultura e lazer são fundamentais para se atingir um envelhecimento ativo. 
Por fim, algumas percepções mais evidentes que emergiram dessa pesquisa e que contribuíram para a perda e/ou diminuição da efetividade do cuidado ao idoso com deficiência física: a deficitária rede de suporte em saúde para os idosos; a escassez de capacitações para as equipes de ESF na área do idoso; a deficiência na formação acadêmica no âmbito da atenção à saúde do idoso e a vigência do modelo biomédico na atenção primária. A principal limitação deste estudo foi a reduzida participação dos profissionais de saúde que não aceitaram participar da pesquisa.

\section{REFERÊNCIAS}

1.Stephens C, Alpass F, Towers A, Noone J, Stevenson $B$. The effects of socioeconomic inequalities of working life on health: implications for an ageing population. New Zealand Journal of Social Sciences Online. 2011;6:73-85. 2.Schoenenberger AW, Stuck AE. Health care for older persons in Switzerland: a country profile. J Am Geriatr. 2006;54:986-90.

3.World Health Organization. International classification of functioning disability and health (ICIDH-2). Geneva (Swi): WHO; 2001.

4.Ministério da Saúde (Br). Secretaria de Atenção à Saúde. Manual de legislação em saúde da pessoa com deficiência. 2ª ed. Brasília (DF): Editora MS; 2006.

5.Secretaria de Direitos Humanos da Presidência da República (Br). Cartilha do Censo 2010 - Pessoas com Deficiência. Brasília (DF): SDH-PR/SNPD; 2012.

6.Ministério da Saúde (Br). Secretaria Especial dos Direitos Humanos. Coordenadoria nacional para a integração da pessoa portadora de deficiência. Acessibilidade. Brasília (DF): SEDH; 2005.

7.Resende APC, Vital FMP. A convenção sobre os Direitos das Pessoas com deficiência comentada. Brasília: Secretaria Especial dos Direitos Humanos; 2008.

8.Instituto Brasileiro de Geografia e Estatística. Censo Demográfico: Brasil, 2010. Rio de Janeiro: IBGE; 2010. 9. Gonçalves LTH, Leite MT, Hildebrandt LM, Bisogno SC, Biasuz S, Falcade BL. Convívio e cuidado familiar na quarta idade: qualidade de vida de idosos e seus cuidadores. Rev Bras Geriatr Gerontol. 2013; 16:315-25. 10.Araújo RSS, Silva AAS, Santos AG, Costa JP. Idosos institucionalizados: perfil social, clínico e funcional. Rev Enferm UFPI. 2014; 3:69-77.

11.Guedes SL. Vidas revisitadas: a reconstrução do passado de idosos em programas gerontológicos. Sociologia
\& Antropologia. 2014; 4:233-55.

12.Lopes MCL, Santos AL, Marcon SS. Convivência familiar com a dependência decorrente do acidente vascular encefálico em hipertensos. Semina: Ciências Biológicas e da Saúde. 2014; 1:75-86.

13.Marin MJS, Santana FHS, Moracvick MYAD. Percepção de idosos hipertensos sobre suas necessidades de saúde. Rev esc enferm USP. 2012; 46: 103-10.

14.Seredynsky FL, Rodrigues RAP, Diniz MA, Fhon JRS. Percepção do autocuidado de idosos em tratamento paliativo. Rev Eletr Enf. 2014; 16:286-96.

15.Veras RP. Prevenção de doenças em idosos: os equívocos dos atuais modelos. Cad Saúde Pública. 2012; 28:1834-40.

16.Corrêa LR. O grupo operativo e a promoção de saúde mental para idosos(as). Revista Saúde e Desenvolvimento. 2013; 3:96-117.

17.Teixeira ER, Barbosa MS, Silva CMC. Trabalhando a transdisciplinaridade na clínica do cuidado em saúde. Rev Enf Profissional. 2014; 1:315:30.

18.Quintana JM, Ferreira EZ, Santos SSC, Pelzer MT, Lopes MJ, Barros EJL. A utilização da Classificação Internacional de Funcionalidade, Incapacidade e Saúde no cuidado aos idosos. Rev Enf Ref. 2014; 1:145-52.

19.Mitre SM, Andrade EIG, Cotta RMM. O acolhimento e as transformações na práxis da reabilitação: um estudo dos Centros de Referência em Reabilitação da Rede do Sistema Único de Saúde em Belo Horizonte, MG, Brasil. Ciênc saúde coletiva. 2013; 18:1893-902 .

20.Polaro SHI, Gonçalves LHT, Alvarez AM. Construindo o fazer gerontológico pelas enfermeiras das Unidades de Estratégia Saúde da Família. Rev esc enferm USP. 2013; 47:160-7

21.Silva KM, Vicente FR, Santos SMA. Consulta de enfermagem ao idoso na atenção primária à saúde: revisão integrativa da literatura. Rev Bras Geriatr Gerontol. 2014; 17:681-7.

22.Kletemberg DF, Padilha MI. Enfermagem gerontológica: a produção do conhecimento na profissão (1970-1996). Rev Gaúcha Enferm. 2013; 34:86-93.

23.Dias AM, Sandri JAV, Cutolo LRA. Saúde Transform Soc. 2012; 3(1): 67-71.

24.Santos I, Guerra RG, Silva LA. Características individuais e clínicas de pessoas idosas com diabetes: investigação temática em oficina sociopoética. Rev enferm UERJ.2013;21:34-40.

25.Tier CG, Santos SSC, Pol MA, Hilgert RM.Condições de saúde dos idosos na atenção primária à saúde. Rev Rene. 2014;15:668-75. 